\title{
Tel Quel à la rue : subjectivation et argumentation dans le discours théorique et dans l'affiche militante de Mai 68
}

Tel Quel in the Streets: Subjectivation and Argumentation in Theoretical Discourse and in Activist Posters during May 68

\section{François Provenzano}

\section{Q OpenEdition Journals}

\section{Electronic version}

URL: http://journals.openedition.org/aad/1897

DOI: $10.4000 /$ aad. 1897

ISSN: 1565-8961

\section{Publisher}

Université de Tel-Aviv

\section{Electronic reference}

François Provenzano, «Tel Quel à la rue : subjectivation et argumentation dans le discours théorique et dans l'affiche militante de Mai 68 », Argumentation et Analyse du Discours [Online], 14 | 2015, Online since 09 April 2015, connection on 23 September 2019. URL : http://journals.openedition.org/aad/ 1897 ; DOl : 10.4000/aad. 1897

This text was automatically generated on 23 September 2019.

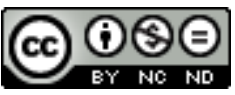

Argumentation \& analyse du discours est mis à disposition selon les termes de la licence Creative Commons Attribution - Pas d'Utilisation Commerciale - Pas de Modification 4.0 International. 


\title{
Tel Quel à la rue : subjectivation et argumentation dans le discours théorique et dans l'affiche militante de Mai 68
}

\author{
Tel Quel in the Streets: Subjectivation and Argumentation in Theoretical \\ Discourse and in Activist Posters during May 68
}

François Provenzano

\section{Introduction : « libérez l'expression »}

1 S'il est une période où les mots d'ordre encourageant l'action collective ont fleuri sous diverses modalités, c'est bien la période qui entoure Mai 68 en France. On peut dire qu'on assiste alors, dans plusieurs secteurs de la production discursive - du plus savant au plus trivial -, à un profond renouvellement des topiques ${ }^{1}$, qui touche à la définition du sujet dans son rapport à l'agir social, et qui continue encore parfois d'imprégner notre imaginaire contemporain. Parmi ces nouvelles topiques transversales, la « liberté d'expression" occupe sans doute une place de choix, presque matricielle, dans la mesure où elle concerne la possibilité et les modalités mêmes de l'activité discursive dans le champ social.

Dans son étude sur Les mots de Mai 68, Maurice Tournier accorde un long article à l'entrée «Liberté » et évoque le slogan «Libérez l'expression », qu'il définit comme une « exigence [...] fortement affichée en opposition à loi, ordre, police, règle » (2007:69). Ce slogan «libérez l'expression» autorise une interprétation précise, qui se distingue singulièrement de la revendication générique de «liberté d'expression »: il s'agirait moins de réclamer pour l'individu les moyens de s'exprimer librement que de libérer le matériau même de la production signifiante. En deçà donc d'une appropriation singulière de la parole et de sa fonction transitive de communication, le slogan viserait 
plutôt l'interdiscursivité, l'ouverture des ressources expressives à une multitude de possibles, et leur libre circulation, indépendante de toute assomption par une instance subjective. On pourrait même dire, en forçant encore un peu l'interprétation, que cette assomption subjective est précisément ce qu'évite le slogan "Libérez l'expression »: l'expression libre, ou plutôt libérée, appelle la dé-prise énonciative, la désidentification du sujet par rapport au message produit, dont l'émergence et la circulation sont rendues au jeu des renvois entre signifiants dans la sémiosis sociale ${ }^{2}$. L'un des tracts d'un comité d'action de Mai 68 invite explicitement à ce jeu interdiscursif :

Relisez cet appel encore et encore. / Devenez-en l'auteur. / Corrigez-le et recopiez-

le. / Diffusez-le à des millions d'exemplaires. / Affichez-le3 .

Or, c'est précisément dans ce type de dés-identification énonciative que le philosophe Jacques Rancière - dont on peut dire qu'il est l'un de ceux qui ont le plus travaillé à éviter la sclérose de l'héritage intellectuel de Mai 68-voit l'occasion d'une subjectivation politique. Tandis que «la police", dit Rancière, est ce qui maintient l'identité entre un lieu, un profil et une fonction pour chaque individu dans la société, tandis que cette police se manifeste comme une «forme d'intervention qui prescrit le visible et l'invisible, le dicible et l'indicible» (2004:211), la subjectivation politique opère à rebours comme un « processus de désidentification ou de déclassification » (ibid. : 119). À cet endroit, Rancière prend comme exemple le fameux slogan de Mai 68 , « Nous sommes tous des Juifs allemands ", pour illustrer ce procès d'émancipation qui, pour forger un sujet politique, renonce précisément à l'expression d'une identification tribale, ségrégative, telle qu'elle relayerait l'assignation identitaire voulue par le discours d'autorité. Le sujet politique se définit ainsi par le ratage qu'il fait subir à la parole du pouvoir, par le jeu qu'il introduit entre les signifiants et les signifiés de cette parole. Ce processus émancipatoire est illustré, à l'extrême, par cette "proposition ultime adressée aux étudiants» (Loyer 2008: 170), dans le tract déjà cité plus haut: "Supprimons-nous : devenons des travailleurs» (ibid.). C'est donc sous cette forme de déprise identitaire que les acteurs de l'époque ont pu fantasmer leur contestation politique de l'autorité.

4 Le parti méthodologique qui sera le nôtre ici consistera à prendre ce fantasme au sérieux pour en examiner les soutiens rhétoriques dans deux discours a priori bien distincts: le genre de la théorie, tel qu'il est investi collectivement, dans le recueil Théorie d'ensemble de Tel Quel paru en 1968, et le corpus des affiches militantes de maijuin de la même année ${ }^{4}$. Nous reviendrons plus loin sur la justification socio-historique de ce rapprochement; disons pour l'heure qu'il nous paraît intéressant, selon une perspective socio-rhétorique, d'éclairer dans une conjoncture précise le fonctionnement d'une topique très générale («libérer l'expression»), qui a pour particularité d'engager l'acte même de sa manifestation en discours. Plaider la libération de l'expression ne peut se faire, en toute cohérence, qu'à partir d'une position qui résulte déjà du procès qu'elle revendique, une position qui, en somme, s'assume comme un refus d'identification sociale pleine et univoque et réalise déjà, en amont, le brouillage des sources énonciatives qu'elle appelle en aval. Comment ce paradoxe argumentatif est-il résolu, ou contourné, dans chacun des sous-corpus considérés? Quelles modulations chacun de ces sous-corpus fait-il subir à la même topique de départ? En somme, comment ce qui est perçu comme un vaste courant doxique propre à l'époque se concrétise dans des formes discursives particulières? 
5 Ces divers ordres de questionnement seront envisagés en plusieurs temps. Après un rappel des éléments de problématisation socio-historique, il s'agira de mettre en lumière ce qui constitue l'argumentaire commun aux diverses contributions individuelles du volume Théorie d'ensemble. Il s'agira ensuite de voir comment cet argumentaire est aussi ce qui fonde l'efficace rhétorique de certaines affiches de Mai 68. Enfin, nous montrerons ce qui distingue l'argumentaire telquelien de l' argumentation des affiches, en particulier en ce qui concerne les effets de subjectivation dont ces discours sont porteurs.

\section{Tel Quel et Mai 68 : éléments de problématisation socio-historique}

6 Si l'on s'intéresse, comme l'a fait Kristin Ross, aux «vies ultérieures" des divers discours qui ont entouré Mai 68, on est frappé de constater les convergences que présentent, dans les modes de réception dont ils ont fait l'objet, l'effervescence théorique et le militantisme politique. Concernant l'éruption urbaine de discours subversifs, Ross résume le tableau en distinguant deux "confiscations » ultérieures : « la version biographique (personnalisation) et la version sociologique» $(2010: 13)$. Soit Mai 68 est retraduit, du point de vue de certains de ses acteurs, comme la geste héroïque de quelques-uns, soit les événements sont catégorisés comme une mutation structurelle étroitement associée à une génération (« la jeunesse ») et à un milieu (« les étudiants »), mus par leurs intérêts particuliers plutôt que par une volonté réellement politique.

7 Ces deux lectures se sont tout aussi bien appliquées à l'avant-garde théorique Tel Quel. Dans les monographies de Philippe Forest (1995) et de Patrick Ffrench (1995), mais aussi dans les discours de Philippe Sollers et de Julia Kristeva eux-mêmes ${ }^{5}$, l'aventure Tel Quel est relue à la lumière des destins personnels de ses leaders et sacralisée par la nostalgie d'un regard rétrospectif, apologétique et individualisant. À l'inverse, dans les travaux de Louis Pinto (1991), de Niilo Kauppi (1994) et, dans une moindre mesure, de Boris Gobille (2005), les positions d'avant-garde sont réduites aux logiques structurelles des différents champs (littéraire, philosophique, universitaire) dans lesquels le groupe a pu capitaliser ses dispositions pour en tirer un maximum de profits symboliques; ici encore donc, la lecture politique est réfutée, puisque ce qui la fonde serait un pur effet de posture : Pinto parle ainsi d'une «parodie de l'intellectuel [...] alliant pédantisme et désinvolture » (1991: 74), tandis que Kauppi insiste sur la «multipositionnalité » du groupe, qui lui assure une "domination intellectuelle efficace » auprès d'un public choisi de jeunes bourgeois (1994: passim) ${ }^{6}$.

8 Que ces massifs socio-discursifs soient reçus par le prisme d'une idéologie collectiviste, ou au contraire individualisante, le résultat demeure donc identique: ces "confiscations", pour reprendre le mot de Kristin Ross, mettent à l'écart le projet politique qui avait pu animer les acteurs de l'époque. Ce projet politique concernait notamment le statut de l'artiste dans la société, aussi bien chez Tel Quel que, cette fois plus spécifiquement, dans l'affichage de rue en Mai 68. La production d'affiches est due à l'Atelier populaire de l'École des Beaux-Arts, constitué par les étudiants et artistes qui décident d'occuper les locaux de l'établissement et de mettre leurs compétences et les machines au service de la contestation populaire. Un des documents rassemblés par 
Alain Schnapp et Pierre Vidal-Naquet dans leur précieux Journal de la commune étudiante témoigne de ce qui anime cette entreprise, dans les termes suivants :

Atelier populaire: oui / Atelier bourgeois: non. [...] Qu'est-ce que la culture bourgeoise? C'est l'instrument par lequel le pouvoir d'oppression de la classe dirigeante sépare et isole du reste des travailleurs les artistes en leur accordant un statut privilégié. [...] Les concepts fondamentaux qui sous-tendent cette action isolatrice qu'exerce la culture sont :

- l'idée que l'art a « conquis son autonomie » [...]

- la défense de la « liberté de création ». La culture fait vivre l'artiste dans l'illusion de la liberté [...] Il n'est pas un travailleur aux prises avec la réalité historique. L'idée de création irréalise son travail. (Schnapp et Vidal-Naquet $1988: 803-804)^{7}$.

Ces thèses et les concepts qui les soutiennent font inévitablement écho au réquisitoire dressé par Tel Quel contre l'idéologie bourgeoise et son traitement de l'art et de la littérature. La proximité des citations est frappante, entre l'extrait précédent et ce passage de l'article de Jean-Louis Baudry, "Écriture, fiction, idéologie », repris dans Théorie d'ensemble :

La double valeur des œuvres - artistique et financière - dépend paradoxalement de la gratuité de leur production. Injustifiables, injustifiées, elles semblent contester les formes, elles ne contestent pas le système. La « liberté » garantit la souveraineté du système, tandis que le système à tous ses niveaux (et d'abord économique) réclame le camouflage de son procès de production. [...] De cette idéologie dépend le statut privilégié de « l'écrivain », apparenté comme « créateur » à Dieu, comme instrument du « verbe » au prêtre, et, comme garant du bon usage de la grammaire et de la légalité de la langue, au magistrat... [...] (ThE : 127-1288).

10 De part et d'autre, il s'agit donc de se libérer de l'illusion de liberté que la société bourgeoise donne à l'artiste, en réservant à son activité ce privilège de la gratuité qui, en réalité, lui dénie la vérité de son inscription historique. De part et d'autre, le " créateur " entend dénoncer (et renoncer à) cette assignation identitaire qui stérilise littéralement son action sur le monde. C'est donc une même contestation de la division sociale bourgeoise du travail qui caractérise la conjoncture ici examinée. Cette critique s'incarne notamment dans l'abolition des frontières entre les catégories " étudiants ", " ouvriers ", " paysans » pour les militants de Mai 68 ; entre celles d'« écrivains » et d'« universitaires » pour les théoriciens de Tel Quel.

11 Outre ces échos communs en surface, qu'en est-il des données socio-historiques effectives quant à l'intersection entre les deux corpus? Le travail a été mené, et de manière très complète, par Boris Gobille (2005), qui démêle tous les enjeux de la «conjoncture de crise » qu'a représenté Mai 68 pour les avant-gardes littéraires du moment, Tel Quel en tête. Inutile de résumer ici l'analyse minutieuse de Gobille, à laquelle nous renvoyons le lecteur ${ }^{9}$; nous nous contenterons de souligner, avec l'auteur, que Mai 68 place en quelque sorte Tel Quel face aux conséquences effectives de ses bravades théoriques : la crise confronte en effet le groupe à l'exigence d'une mise en œuvre concrète de ses mots d'ordre sur la «mort de l'auteur» et sur "l'écriture révolutionnaire ", entre autres. Cette mise à l'épreuve révèle, selon Gobille, le profond «malthusianisme» qui anime le groupe, qui « exceptionnalise l'accès à la grandeur littéraire» (ibid. : 51). À l'inverse, c'est le CAEE (Comité d'action étudiants-écrivains révolutionnaires) qui insiste sur la dépersonnalisation de l'écrivain en contexte révolutionnaire, sur l'anonymat du scripteur révolté et qui, avec le collectif de Faye, « capt[e] une légitimité révolutionnaire alternative au ralliement orthodoxe au PCF, et plus rentable du point de vue symbolique et auprès du lectorat étudiant » (ibid. : 49) ${ }^{10}$. 
12 En outre, il faut encore rappeler, avec Gobille (2005:49) et Loyer (2008:34-38), que les pratiques d'écriture spontanée puisent, dans leur forme, davantage aux fonds surréalistes et situationnistes, souvent bien antérieurs, qu'au "théoricisme » contemporain.

Or, ce sont justement ces dimensions formelles qui nous intéressent ici, dont l'objectif pourrait être ainsi de donner une sorte de contre-éclairage rhétorique aux analyses socio-historiques déjà menées sur la même conjoncture. Les travaux de Ross et Gobille déjà cités mais aussi celui, encore plus proche de notre propos et tout à fait stimulant, de Trebitsch (2000) décrivent en effet dans le détail toute l'infrastructure historique et socio-discursive, dans laquelle s'inscrit cette topique libératoire, toute l'importance qu'elle prend à l'intersection des sphères savantes et profanes, mais sans jamais entrer vraiment dans l'analyse des productions discursives elles-mêmes ${ }^{11}$, en tant qu'elles entendent participer, au plus proche de leur matérialité, des enjeux politiques du moment. Les seuls éléments saillants, par ailleurs fréquemment relayés, concernent le symbolisme convoqué par les discours militants (1789, la Résistance ; voir Loyer 2008 : 272), la "spontanéité » ou "l'oralité » des formes écrites (Loyer 2008: 287), le détournement phraséologique ( $\mathrm{du}$ communisme, de l'autoritarisme), ou le caractère poétique des slogans («L'imagination au pouvoir »). C'est sans doute à Roland Barthes (1968) et à Michel de Certeau (1968), en exacts contemporains du corpus concerné, que l'on doit le regard le plus aiguisé sur ce qu'ils ont tous deux nommé cette " prise de [la] parole $»^{12}$. Ce sont précisément les mécanismes énonciatifs et rhétoriques de cette prise qu'il nous importe d'éclairer, en évitant aussi d'isoler les seuls slogans des supports iconiques qui les portaient concrètement dans l'espace public $^{13}$. Le choix de cette perspective devrait nous permettre de mieux comprendre en quoi ces discours de rue contrastent avec le discours collectif de Tel Quel, malgré plusieurs convergences topiques de surface.

\section{La topique émancipatoire de Tel Quel}

Au-delà de la diversité des contributions au recueil Théorie d'ensemble, quant au ton et quant aux thèses défendues, on peut identifier globalement un argumentaire commun, qui consiste à condamner deux aspects majeurs du fonctionnement sémantique du texte littéraire dans la société bourgeoise: la représentation mimétique et l'expressivité subjective ${ }^{14}$. L'idée d'une « œuvre» originale produite par la conscience d'un « auteur » singulier pour délivrer un «message » sous la forme d'un signifié plein et univoque, est sévèrement dénoncée comme un asservissement de la littérature à l'idéologie bourgeoise, capitaliste et consumériste. La théorie telquelienne du texte est en effet indexée sur les thèses de l'économie politique marxiste, dont la grille de lecture repose sur le couple "production » - « exploitation ». La société bourgeoise ne demande qu'à consommer et à accumuler du signifié, et exploite ainsi les forces de production signifiante à l'œuvre dans l'écriture, tout en réclamant le masquage du procès de productivité textuelle. Ce procès, à la fois mis au jour et incarné par l'activité théorique qu'on porte à son endroit, ne peut par définition être assigné à aucune individualité créatrice en amont, ni déboucher sur aucune signification pleine en aval. Il est pur jeu de renvois entre signifiants, qui portent chacun la trace de leurs inscriptions historiques respectives. En cela, l'écriture, inséparablement pratique et théorie de cette pratique, est le lieu possible de la révolution, puisqu'elle conteste les 
identités énonciatives et les signifiés esthétiques que l'ordre social dominant produit par le biais d'un "protocole rhétorique " imposé. L'écriture est une subversion de ce protocole représentatif et subjectif, à la fois parce qu'elle le dénonce comme une censure et parce qu'elle lui substitue une intertextualité généralisée comme trace sensible du procès historique de production matérielle du sens.

Nous n'avons fait ici que résumer, sans entrer dans le détail des références et des citations, les traits saillants d'un argumentaire sans doute plus nuancé. Il importe simplement de mettre en évidence une certaine topique culturelle, qui constelle les différents textes du recueil sous la forme d'un lexique de la lutte de libération : il s'agit de recenser les " "coups" portés à l'idéologie simple et de la propriété d'expression » (ThE : 404), de «nous libérer [...] par rapport à l'entassement archéologique de notre culture» (ibid.: 405); on pointera également les mots et expressions tels que " contestation massive», «censure ", « répression", «asservissement", "impérialisme de notre culture », "attaque en règle», "renverser» (ibid.: 67-79), " urgence de détériorer", "exige que l'on contribue massivement à l'agonie » (225, 227), « condamnation ", « répression ", « soumission », « contestation incessante » (127-147), «aliénation", «enfermement », « empêchement» (94-115), " transgression ", « forces répressives » (354-364) - toutes expressions qui, au-delà des auteurs singuliers qui les portent (Philippe Sollers, Denis Roche, Marcelin Pleynet, JeanLouis Beaudry), s'indexent sur une topique commune: l'appel à une lutte émancipatoire sur le terrain de la production culturelle.

Cette topique omniprésente est par ailleurs bien alimentée par le présent des auteurs : c'est « notre culture » qui exploite, censure, réprime ; c'est donc elle qui concentre le lieu et le moment de la lutte, c'est elle dont il faut se dé-prendre par une subversion des conventions de production et de circulation du sens.

Cette subversion, en tant que refus des catégorisations sémiotiques qui assurent l'homogénéité du code d'expression et la transparence des messages subjectifs et représentatifs qu'il produit, se manifeste volontiers chez Tel Quel par les hybridations intertextuelles et, singulièrement, intersémiotiques. Les idéogrammes chinois sont pour Sollers une «force graphique de base sur laquelle vient se briser l'écriture phonétique " (ThE: 77), la référence aux plaques photographiques joue comme une iconisation de l'écrit, dans le projet poétique de Denis Roche, de même que pour JeanLouis Baudry le modèle du hiéroglyphe "insiste sur la corporéité du matériel signifiant» (ibid. : 132). Marcelin Pleynet explique cette fascination pour la trace graphique par un souci de faire du " déchiffrement » le mode de lecture qui " contrarie la lecture de convention » (105-106). Se libérer de l'exploitation sémiotique bourgeoise, c'est donner à voir, littéralement, la concaténation de plusieurs couches sémantiques, la «mobilité des surfaces textuelles fragmentaires" (143), là où l'idéologie logocentrique rend «aveugle » aux forces de transformation à l'œuvre dans le travail scriptural (356).

\section{L'affiche comme feuilletage énonciatif et sémiotique}

On comprend à cet endroit la transition qui s'offre à nous vers le discours des affiches militantes. Définies par l'association (sous diverses modalités) du verbal et du visuel et par la polyphonie qui en résulte, ces affiches seraient comme l'application concrète de l'argumentaire telquelien. Le message politique qu'elles communiquent explicitement 
par leurs signifiés ("Nous sommes le pouvoir ", "Oui usines occupées », «À nous de parler ", etc.) serait supporté par un propos révolutionnaire second, ou plutôt justement premier et plus fondamental, plus profond, dans la mesure où il toucherait à la manière même de manipuler les signifiants. La libération qui se joue là est une réappropriation des codes et des supports de la communication publique, qui s'effectue par un renversement complet des normes énonciatives qui régissaient l'usage de ces codes et de ces supports. L'affiche, en Mai 68, ne suppose pas d'être prise en charge par une autorité institutionnelle (correspondant à un lieu et à une fonction) mais se saisit de tout lieu pour faire fonction d'affiche; son contenu n'est pas un message clos transitant d'un énonciateur particulier à un énonciataire visé, mais apparaît plutôt comme le fragment d'une expérience collective en cours, qui ne cesse de nourrir en retour la proposition formelle que constitue l'affiche; enfin, cette forme de l'affiche se rend lisible et trouve son efficace par le biais d'un travail de déchiffrement, de dépistage des couches sémantiques, qui contrarie l'exigence conventionnelle d'univocité.

Ce feuilletage du sens peut prendre des dimensions multiples. Dans sa version minimale, il consiste en une mise en relation de deux (ou davantage) signifiants linguistiques par le biais d'une parenté phonique - c'est banalement le procédé de la rime - qui produit en retour une ré-interprétation des signifiés. C'est un procédé typique de la rhétorique militante, qu'on trouve par exemple dans les fameux slogans "CRS / SS » ${ }^{15}$ ou «Civic Indic Flic». Selon la formule jakobsonienne, le jeu sur la matérialité du langage invite à une projection de l'axe syntagmatique sur l'axe paradigmatique et, en l'occurrence, à une mise en équivalence des réalités désignées. En jouant a contrario, ce procédé de rapprochement de signifiants phoniques - souvent doublé d'un apparentement graphique - peut souligner un contraste entre deux concepts, comme dans « Flins pas Flics ${ }^{16}$.

La libération du signifiant se fait plus sensible lorsqu'elle invite à connecter, toujours au sein d'une même affiche, le verbal et l'iconique. Cette connexion peut être assez simple, comme dans le cas de l'illustration 1 , où le tracé du signe graphique (la lettre « $\mathrm{i}$ ») est remotivé par son intégration dans l'icône de l'usine ${ }^{17}$. Pragmatiquement, dans le contexte des grèves de Mai 68 , cette interaction verbo-iconique fonctionne comme une argumentation intersémiotique, où l'affirmation verbale (" oui usines occupées ») apparaît comme nécessairement appelée par le graphisme de l'objet sur lequel elle porte. Le signe de l'usine apparaît d'ailleurs dans bien d'autres affiches, comme le support possible de multiples significations, plus ou moins génériques ou conjoncturelles ${ }^{18}$. 


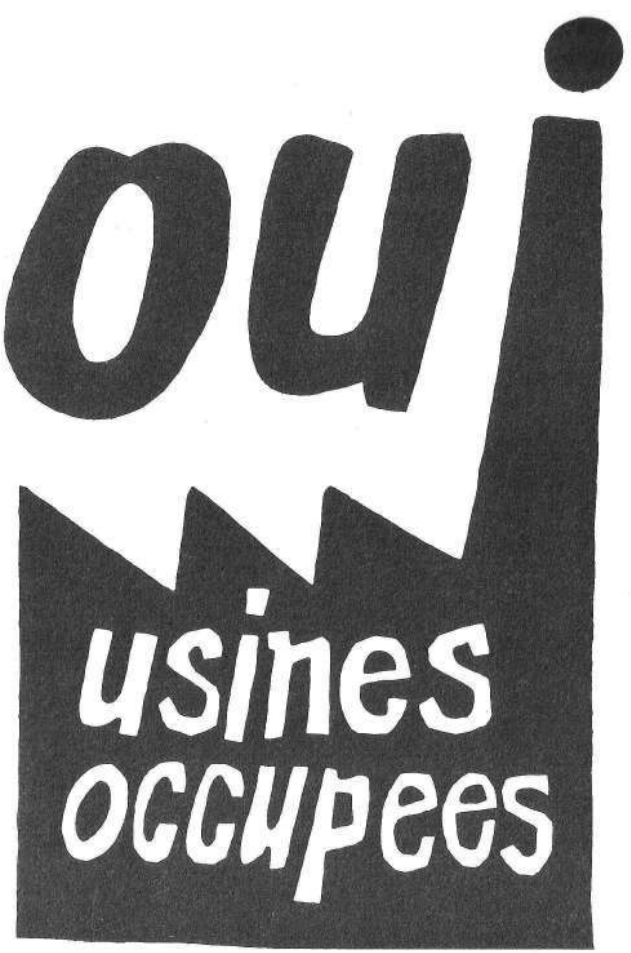

Illustration 1 : Oui usines occupées

Source : Gasquet, Vasco. 2007. 500 affiches de mai 68 (Bruxelles : Aden), p. 189

Un autre procédé de manipulation iconique est à l'œuvre dans l'affiche montrant le lion, symbole de la marque automobile Peugeot, aux prises avec un lutteur (ill. 2): détournée du logo de marque auquel elle était attachée initialement, l'image du lion est ré-iconisée, et cette remotivation du signe visuel rejaillit sur le sémantisme du mot «lutte » du slogan, qui connaît un défigement et retrouve une densité qu'avait pu lui faire perdre sa répétition dans un grand nombre d'occurrences dans les discours de l'époque. Dans ce cas comme dans le précédent, les composantes linguistiques et iconiques de l'affiche entrent en relation pour faire converger leurs contenus sémantiques respectifs. 


\section{LA LUTIE CONTINUE}

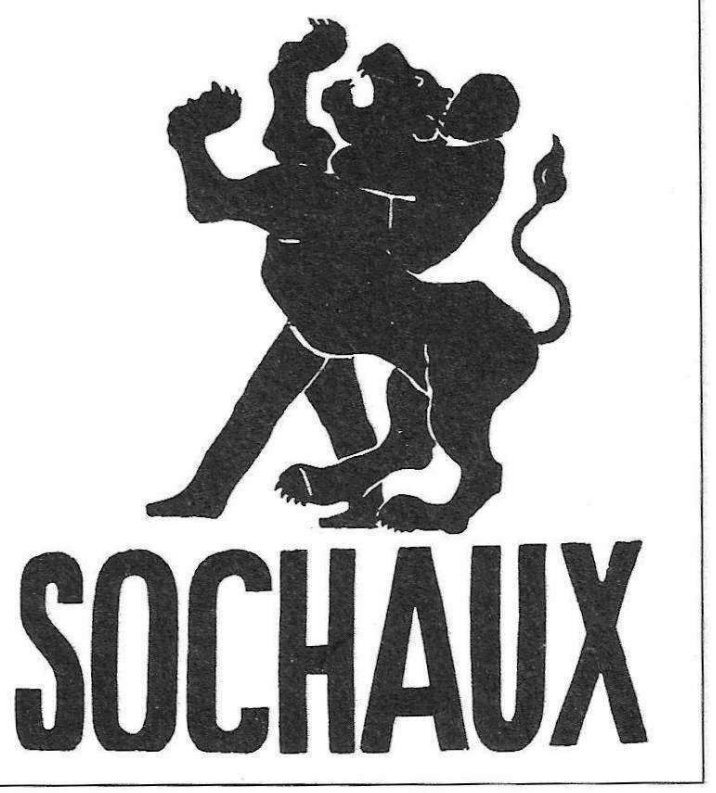

Illustration 2 : La lutte continue

Source: Gasquet, Vasco. 2007. 500 affiches de mai 68 (Bruxelles : Aden), p. 66

Mais le rapport entre texte et image peut aussi jouer sur la dysphorie, plutôt que sur le renforcement sémantique ${ }^{19}$. Dans les illustrations 3-6, l'énoncé verbo-iconique manque d'homogénéité sémantique interne et invite à opérer ce que Klinkenberg (2008) nomme une "accommodation ». L'image affirme quelque chose que le texte ne dit pas, voire propose un sens contraire à ce que le texte dit, et oblige ainsi à réinterpréter le fragment verbal figé, à en creuser la signification au-delà de l'effet de reconnaissance immédiate qu'il peut provoquer lorsqu'il est pris isolément. Le syntagme « retour à la normale » (ill. 3) renvoie de prime abord au discours de l'autorité (éventuellement relayé par les médias); en tant que séquence linguistique, il peut éventuellement être lu déjà de manière ironique, comme le triste constat d'une soumission consensuelle. Or son interaction avec l'image des moutons renvoie ce fragment à une seule énonciation possible, celle de l'autorité, pour souligner par le contraste iconique ce que recouvre réellement cette formule d'apaisement. Les cas d'allotopie ${ }^{20}$, et les opérations d'accommodation rhétorique qu'elles convoquent, sont sans doute plus flagrants encore dans les ill. 4-6, où les composantes iconiques apparaissent comme des surénonciateurs (Rabatel 2009) par rapport aux composantes verbales, dont elles appellent le défigement sémantique. 


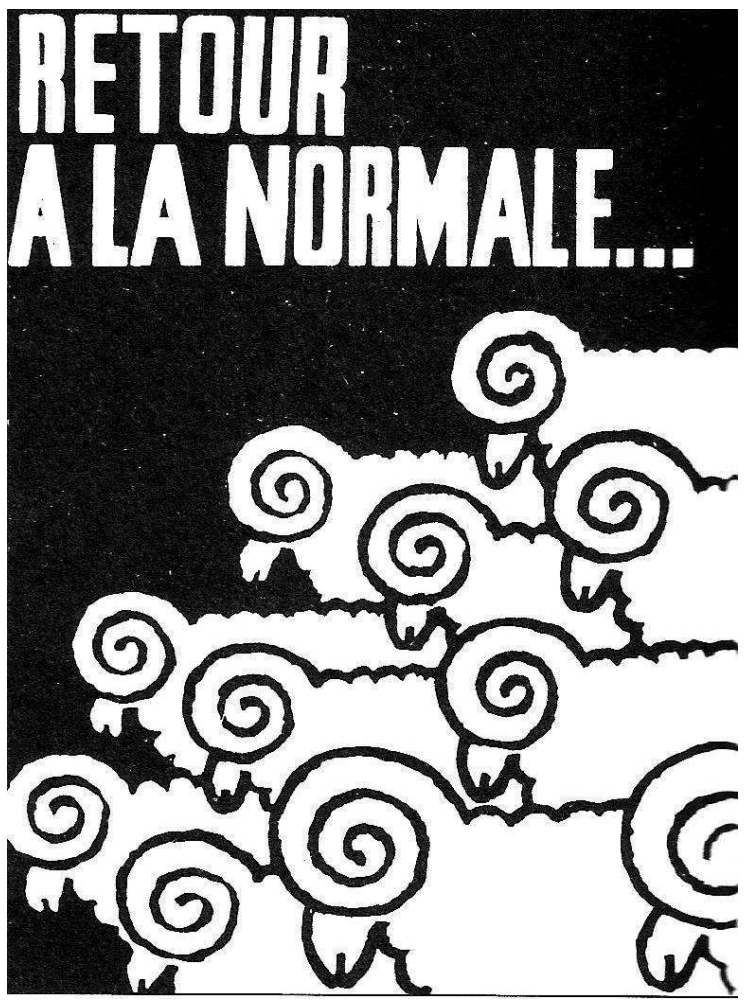

Illustration 3 : Retour à la normale

Source : Gasquet, Vasco. 2007. 500 affiches de mai 68 (Bruxelles : Aden), p. 22

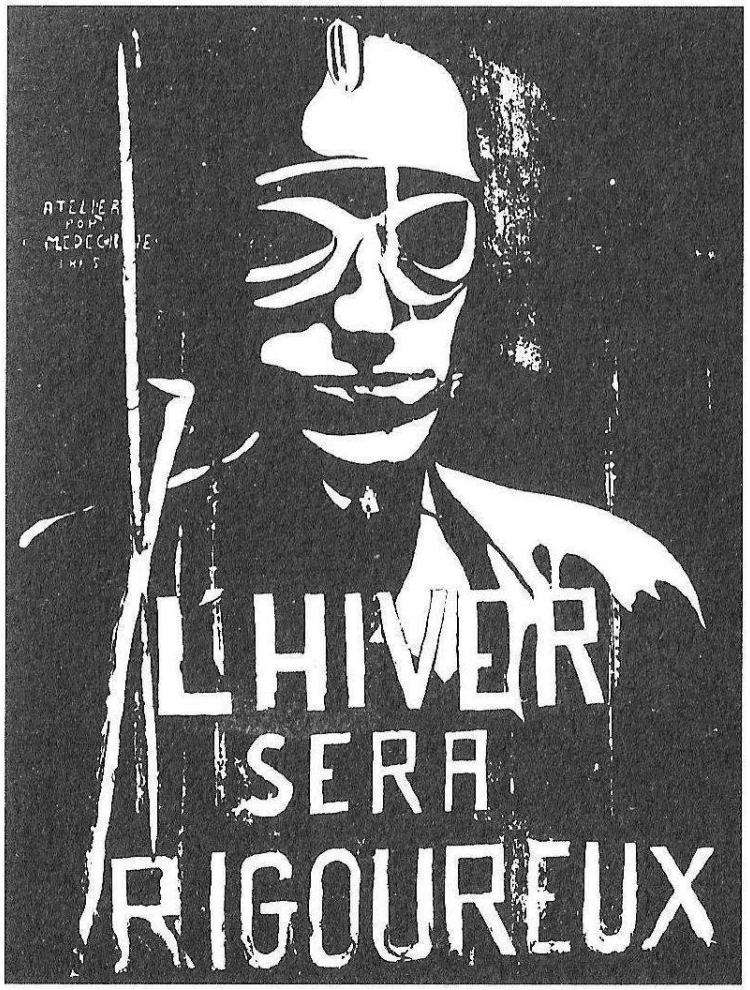

Illustration 4 : L'hiver sera rigoureux

Source: Gasquet, Vasco. 2007. 500 affiches de mai 68 (Bruxelles : Aden), p. 57 


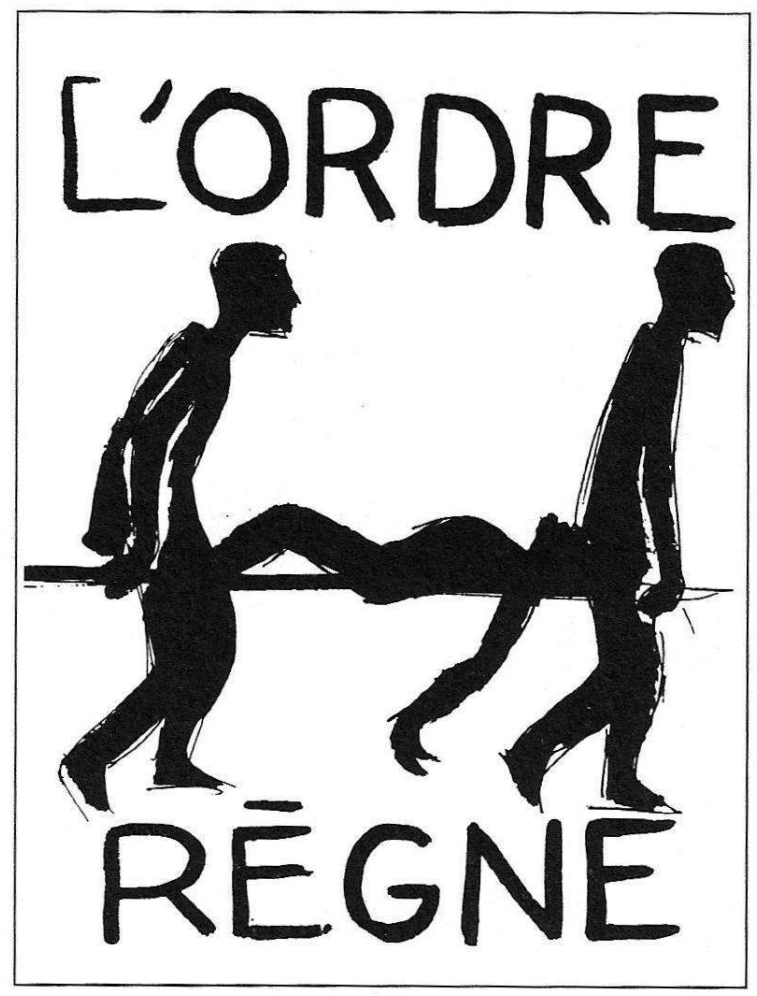

Illustration 5 : L'ordre Règne

Source : Gasquet, Vasco. 2007. 500 affiches de mai 68 (Bruxelles : Aden), p. 48

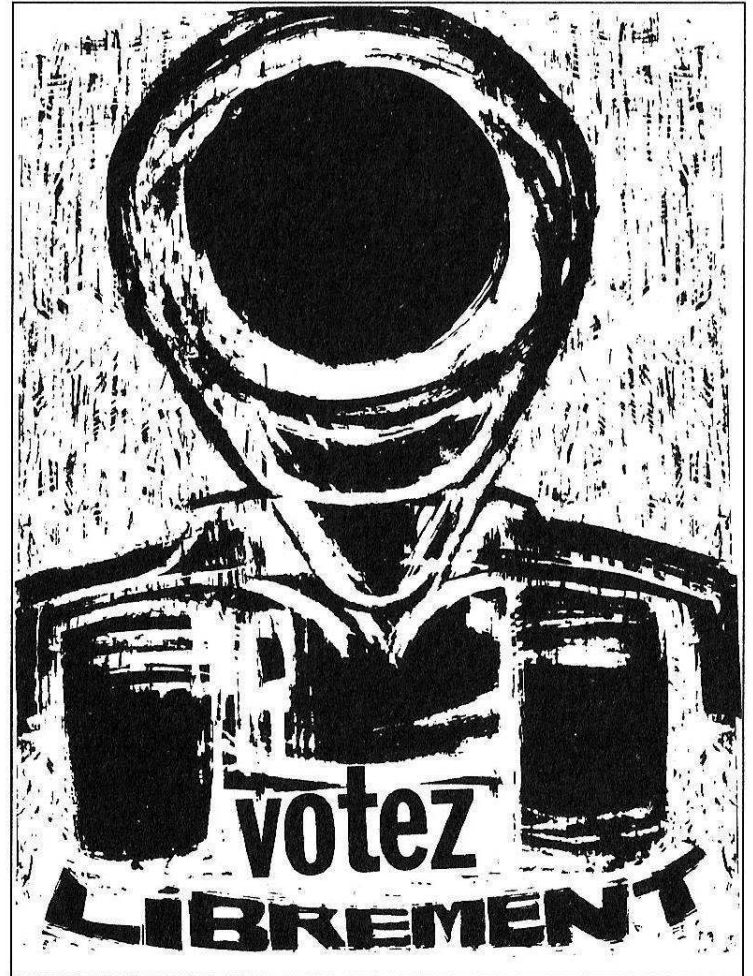

Illustration 6 : Votez librement

Source: Gasquet, Vasco. 2007. 500 affiches de mai 68 (Bruxelles : Aden), p. 57 
23

hypothèse veut que c'est à ce mode particulier de lecture et d'appropriation que les concepteurs comme les lecteurs des affiches de Mai 68 ont pu attacher l'essentiel de leur acte subversif et libératoire ${ }^{21}$. En effet, c'est bien à une forme de connivence énonciative qu'invite ce type de dispositif, proche en cela du fonctionnement de l'ironie : puisque le sens global résulte bien d'une décision de lecture, d'un processus interprétatif appelé par cette décision et du partage des codes culturels qui soutiennent ce processus, ce sens est fortement co-énoncé ; mises bout à bout, ces expériences de co-énonciation constituent potentiellement le ciment discursif d'une "communauté culturelle » qui s'éprouve comme telle ${ }^{22}$.

reviendrons plus loin sur la question des sources énonciatives - car il faut malgré tout se demander "qui parle?" dans l'affiche, qui assume le montage qu'elle réalise ?-, mais achevons avant cela le parcours des différents types de feuilletage sémiotique avec son stade ultime: outre la connexion, au sein de l'affiche, des signifiants verbaux entre eux ou des signifiants verbaux avec les signifiants iconiques, l'affiche de Mai 68 peut s'inscrire et se lire, en tant que textualité matérielle et historique, en rapport avec d'autres textualités, voire d'autres réalités, matérielles et historiques elles aussi. Ce sont là sans doute les argumentations les plus puissantes visuellement, car elles donnent à voir, littéralement, un autre visage de l'histoire en cours, en lui surimposant des signifiants prélevés apparemment en-dehors d'elle, mais agissant dans l'affiche comme des révélateurs d'une profondeur insoupçonnée de l'événement, sur les plans chronologique, idéologique, voire carrément pragmatique.

L'exemple le plus clair de ce procédé est fourni par le fameux portrait de De Gaulle masquant Hitler (ill. 7), avec une reprise des signifiants typiques du nazisme détournés vers le présent de l'affiche (comme le bandeau au bras où figure le symbole gaulliste plutôt que la croix gammée). L'image syncrétise deux strates historiques et deux contextes culturels : l'Allemagne nazie et la France gaullienne. En les syncrétisant, elle invite du même coup à inscrire dans une même série idéologique ce qui, dans l'encyclopédie commune, apparaissait comme des pôles antagonistes : le totalitarisme et la Résistance. Il faut relever également que la "fonction indexicale " (Klinkenberg 2008) remplie par la bordure noire entourant le personnage représenté consiste à clôturer l'espace de la représentation et à le séparer de celui du spectateur urbain, qui constitue ainsi un troisième niveau de pertinence. Or, cette clôture est ici traversée par le bras du personnage s'avançant hors du cadre du portrait: non seulement Hitler apparaît sous De Gaulle, mais il cesse d'être une pure image pour s'animer d'une vie potentiellement extra-iconique. 


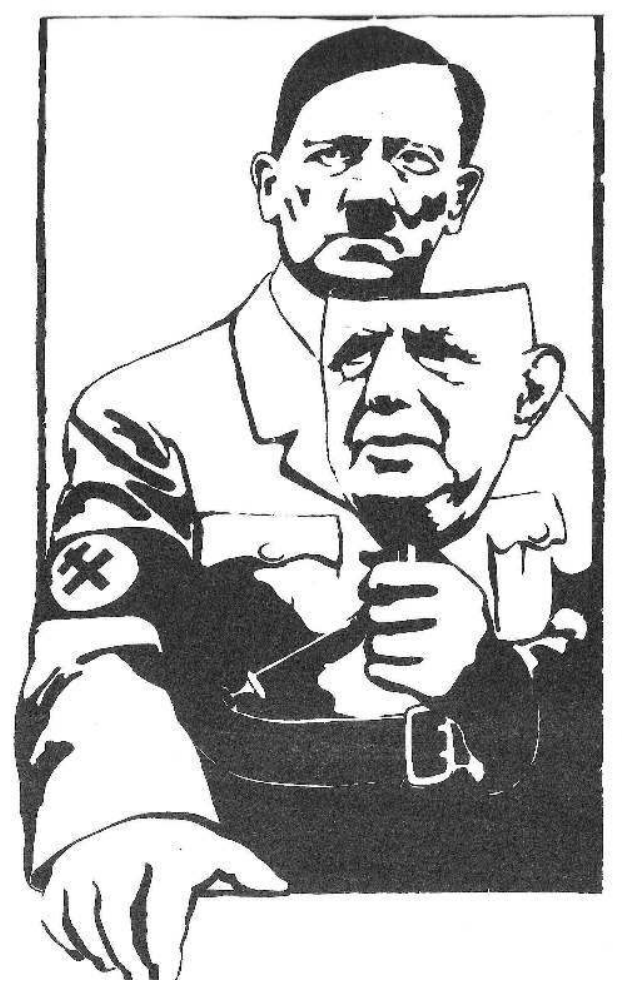

Illustration 7 : De Gaulle masquant Hitler

Source : Gasquet, Vasco. 2007. 500 affiches de mai 68 (Bruxelles : Aden), p. 117

On trouve un autre procédé de syncrétisme historique à l'œuvre dans une affiche reproduisant formellement l'affiche qui fait suite à l'Appel du 18 juin 1940 (ill. 8), mais en l'actualisant selon les enjeux des luttes de 1968 (ill. 9). L'intertexte historique n'est donc pas simplement importé, mais il est donné à voir à travers une série de transformations formelles (la plus notable étant sans doute celle de "France » en « Rage » dans la formule finale). Celles-ci portent trace, littéralement, de la distance historique qui à la fois sépare et unit la France de 1940 et celle de 1968. 


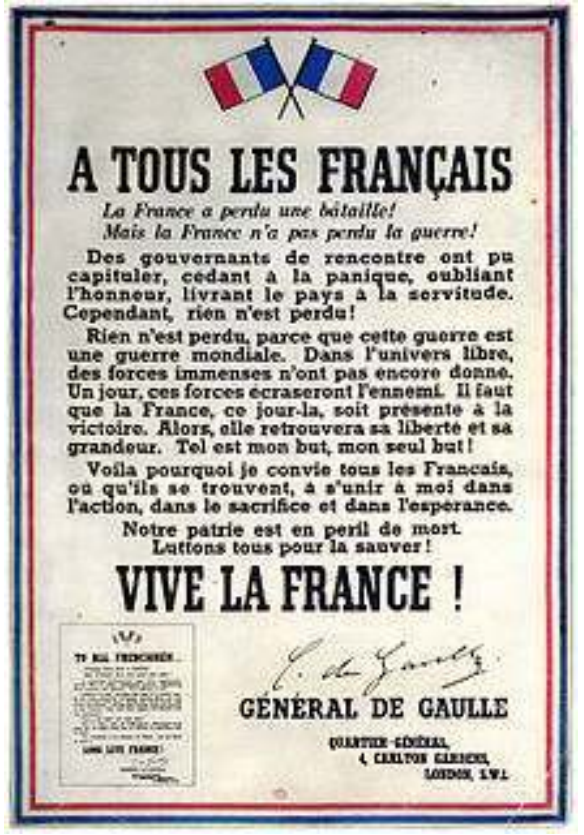

Illustration 8 : Appel du 18 juin 1940

Source : Source : Fondation Charles-de-Gaulle

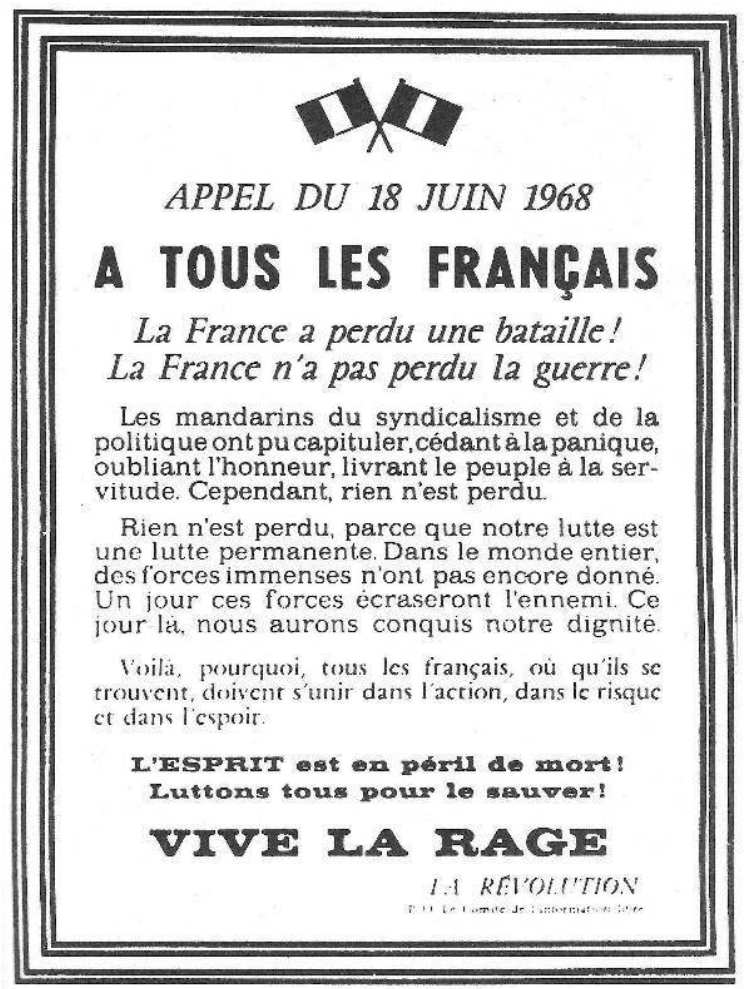

Illustration 9 : Appel du 18 juin 1968

Source: Gasquet, Vasco. 2007. 500 affiches de mai 68 (Bruxelles : Aden), p. 14

L'exemple ultime de ces cas où l'affiche sort d'elle-même pour manifester l'inscription historique de sa textualité est sans doute fourni par cette représentation du pavé de rue comme bulletin de vote (ill. 10). D'un point de vue strictement limité à la syntaxe verbo-iconique (Klinkenberg 2008), le déictique démonstratif «Voici» renvoie bien, tout simplement, au composant iconique qui figure juste sous lui dans la composition 
de l'affiche. Mais il se trouve que des considérations sémantiques et pragmatiques peuvent introduire une ambiguïté sur le caractère interne ou externe de cette référence déictique. D'une part, les composants verbaux ("bulletin de vote») et iconique (l'image du pavé) mis en relation sont radicalement allotopes; d'autre part, cette allotopie est corrigée pragmatiquement par des éléments extérieurs à l'énoncé de l'affiche : le pavé de rue et son emploi très concret dans les luttes urbaines devient ce que focalise réellement l'affiche, amenant le réel le plus matériel à corriger l'énoncé linguistique (le «bulletin de vote » reçoit un sens qui est, davantage qu'une métaphore, un appel à la lutte active sur la scène urbaine de Mai 68).

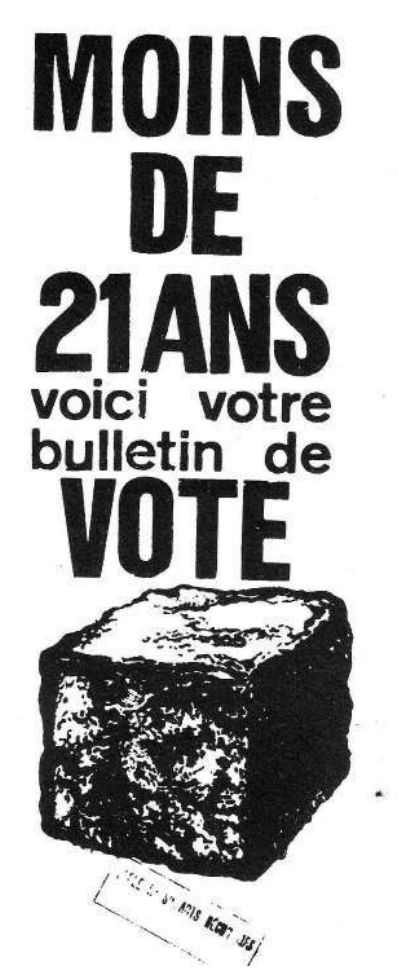

Illustration 10 : Voici votre bulletin de vote

Source : Gasquet, Vasco. 2007. 500 affiches de mai 68 (Bruxelles : Aden), p. 23

\section{Une politique de l'énonciation}

Tous ces éléments que nous venons de mettre en évidence dans le fonctionnement de l'affiche peuvent être compris à la lumière de l'argumentaire telquelien contemporain sur l'écriture textuelle et son potentiel révolutionnaire. Le dernier exemple évoqué (celui du pavé) et la question non résolue de la source du montage énonciatif nous poussent cependant à amender cette première lecture, pour comprendre ce qui dans l'affiche de Mai 68 excède l'argumentaire de Tel Quel et éclairer ainsi, d'un point de vue rhétorique, le constat socio-idéologique dressé notamment par Boris Gobille quant aux divergences entre l'avant-garde de Sollers et le mouvement étudiant.

En schématisant les choses, nous pourrions dire que le discours de Tel Quel dans Théorie d'ensemble se limite à être un argumentaire, plutôt qu'une argumentation ; c'est-à-dire une déclaration d'intention, un programme, un exposé des positions et des principes propres à un ensemble défini d'acteurs, plutôt que la démonstration d'une vérité face 
au constat d'un tort subi, débouchant sur la constitution d'une communauté de sujets politiques (selon les mots de Jacques Rancière 2004).

Cet argumentaire telquelien est en effet produit par des théoriciens bien identifiables, soucieux d'ailleurs de garantir leur identification par le recours à divers types de cautions légitimantes - passons sur la présentation matérielle de l'ouvrage, qui multiplie les signes de personnalisation des discours -, et ne vise rien d'autre que produire des sujets théoriciens, c'est-à-dire forcer l'identification des destinataires aux destinateurs du discours. Les nombreux injonctifs qui émaillent les discours (pensons au titre de Marcelin Pleynet "La poésie doit avoir pour but») participent de cette normativité assumée par le genre de la théorie, seule forme possible de libération des formes de répression bourgeoises.

La rhétorique de l'affiche de Mai 68 déborde singulièrement le cadre de cet argumentaire pour prétendre réaliser cette fois une argumentation spécifique, c'est-àdire l'occasion d'une subjectivation politique dans le cadre du traitement d'un tort, d'une démonstration d'une "faille logique " dans «la mise en acte de l'égalité » (Rancière 2004 : 119-120).

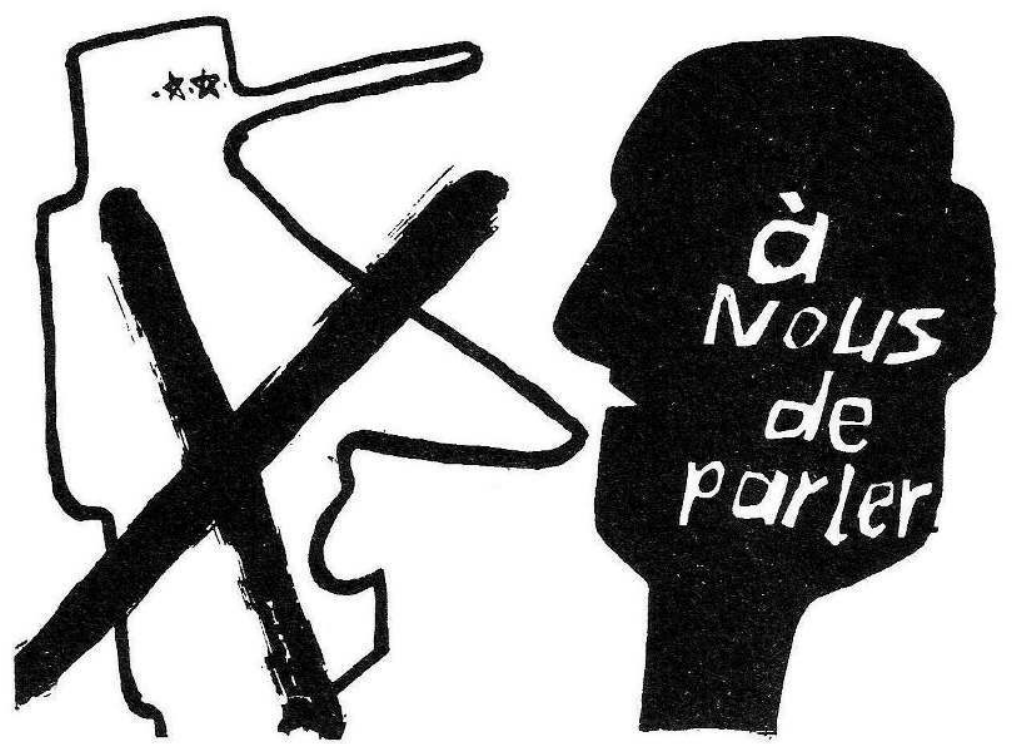

Illustration 11 : À nous de parler

Source : Gasquet, Vasco. 2007. 500 affiches de mai 68 (Bruxelles : Aden), p. 22 


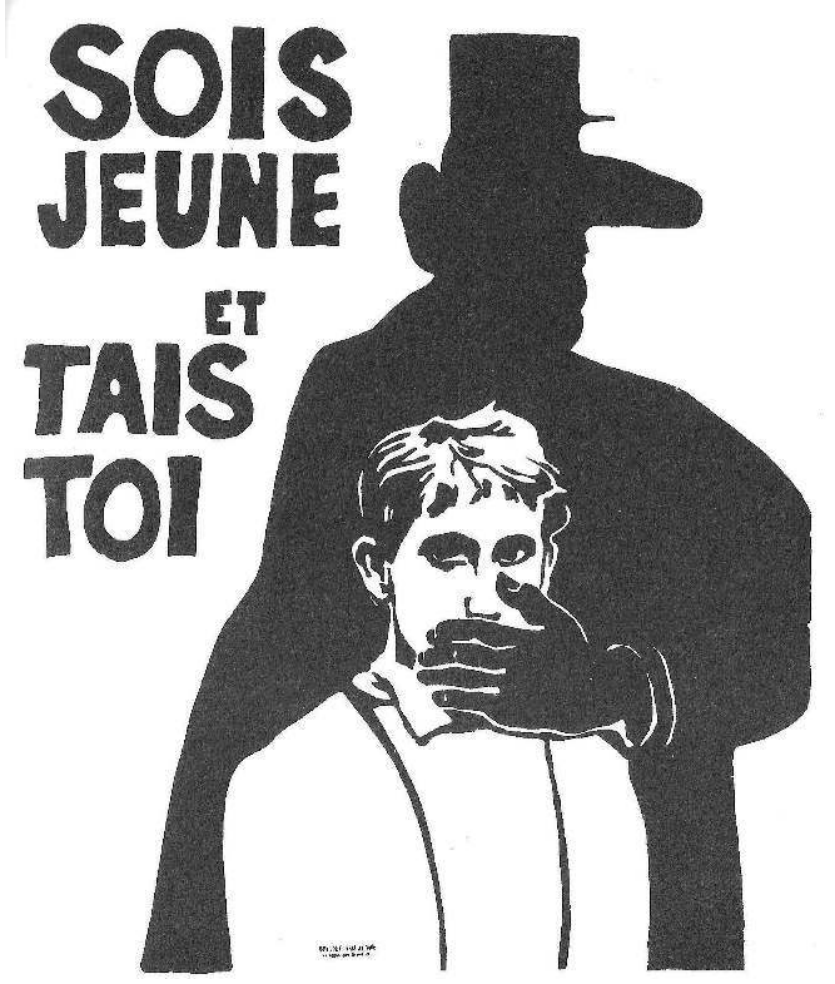

Illustration 12 : Sois jeune et tais-toi

Source : Gasquet, Vasco. 2007. 500 affiches de mai 68 (Bruxelles : Aden), p. 101

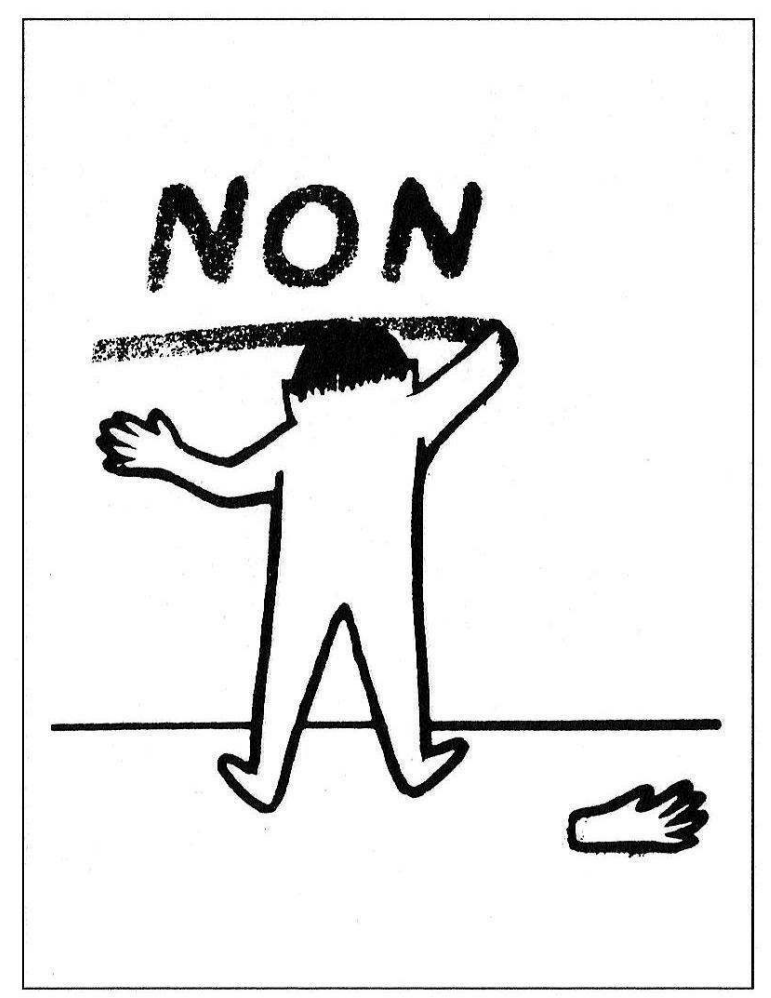

Illustration 13 : Militant à la main coupée écrivant « Non »

Source: Gasquet, Vasco. 2007. 500 affiches de mai 68 (Bruxelles : Aden), p. 54 
Pour ce qui nous intéresse ici, cette égalité revendiquée est celle de la parole, de la production verbale elle-même. De nombreuses affiches thématisent cette police qui s'exerce sur l'activité signifiante: "À nous de parler", "Sois jeune et tais-toi ", l'illustration montrant un militant à la main coupée écrivant « Non » sur le mur, de son propre sang (ill.11-13). Elles thématisent aussi, de manière réflexive, la réponse formulée à cette force de censure: des affiches telles que «La police s'affiche aux Beaux-Arts », « Toute la presse est toxique », « La grève continue » ou « ...ils profitent » (ill. 14-17) mettent en scène l'acte même d'écriture/lecture subversif qui invite à lire le corpus entier des affiches en tant que réappropriation des formes signifiantes par la communauté, et donc qui fantasme la constitution de cette communauté en sujet politique. Ce qui frappe dans ces mises en scène réflexives, c'est le refus de proposer une identification singularisante à la source énonciative, une typification de cette source en tant que profil ${ }^{23}$. La source énonciative est en effet toujours représentée $a$ minima, sans attributs (par contraste avec les caricatures de De Gaulle ou du CRS casqué), en fusion avec le lieu ou le support même de son expression (l'usine ou la banderole), ou encore tout entière absorbée par le geste même de l'inscription par le corps, comme le montre au plus haut degré l'illustration 13.

\section{LA POLLEE S'AFFICHE AUX BEAUX ARTS

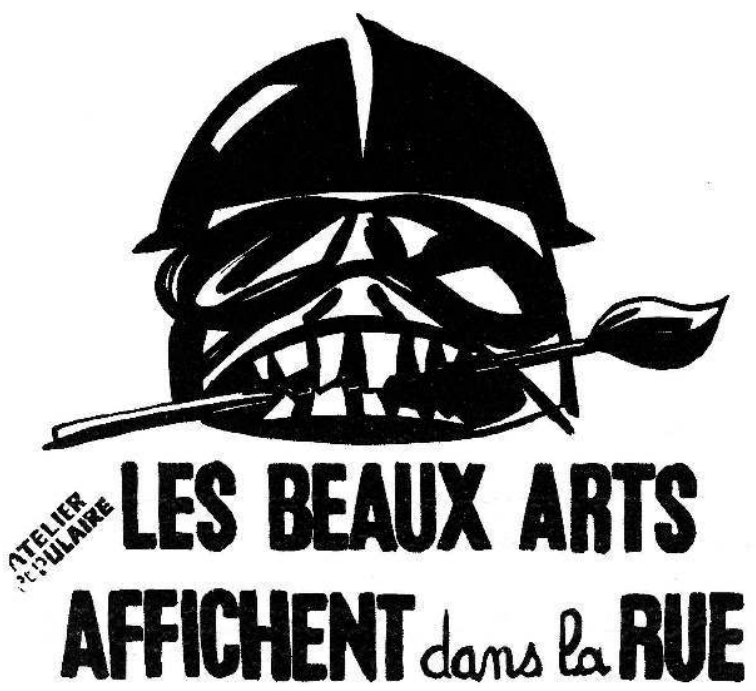

Illustration 14 : La police s'affiche aux Beaux-Arts

Source : Gasquet, Vasco. 2007. 500 affiches de mai 68 (Bruxelles : Aden), p. 32 


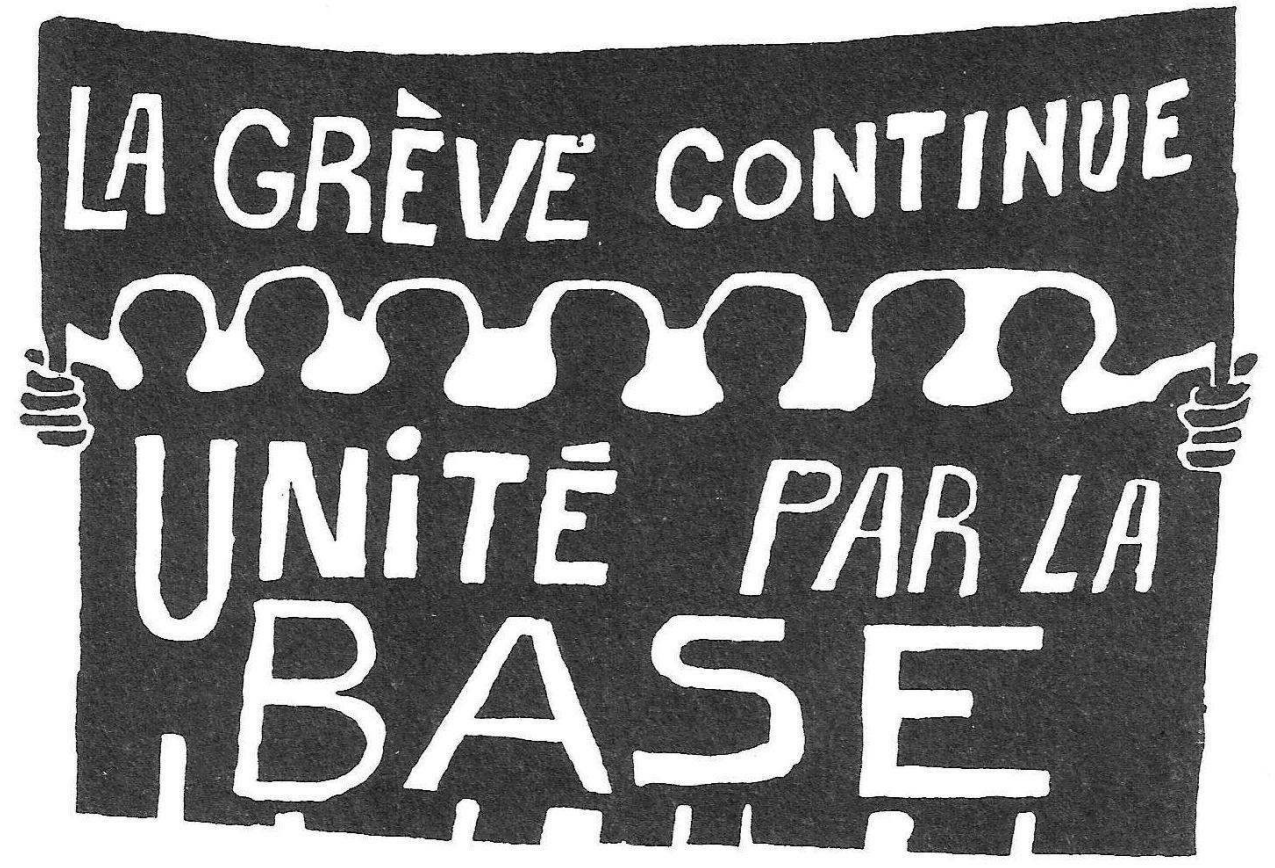

Illustration 15 : La grève continue

Source : Gasquet, Vasco. 2007. 500 affiches de mai 68 (Bruxelles : Aden), p. 194

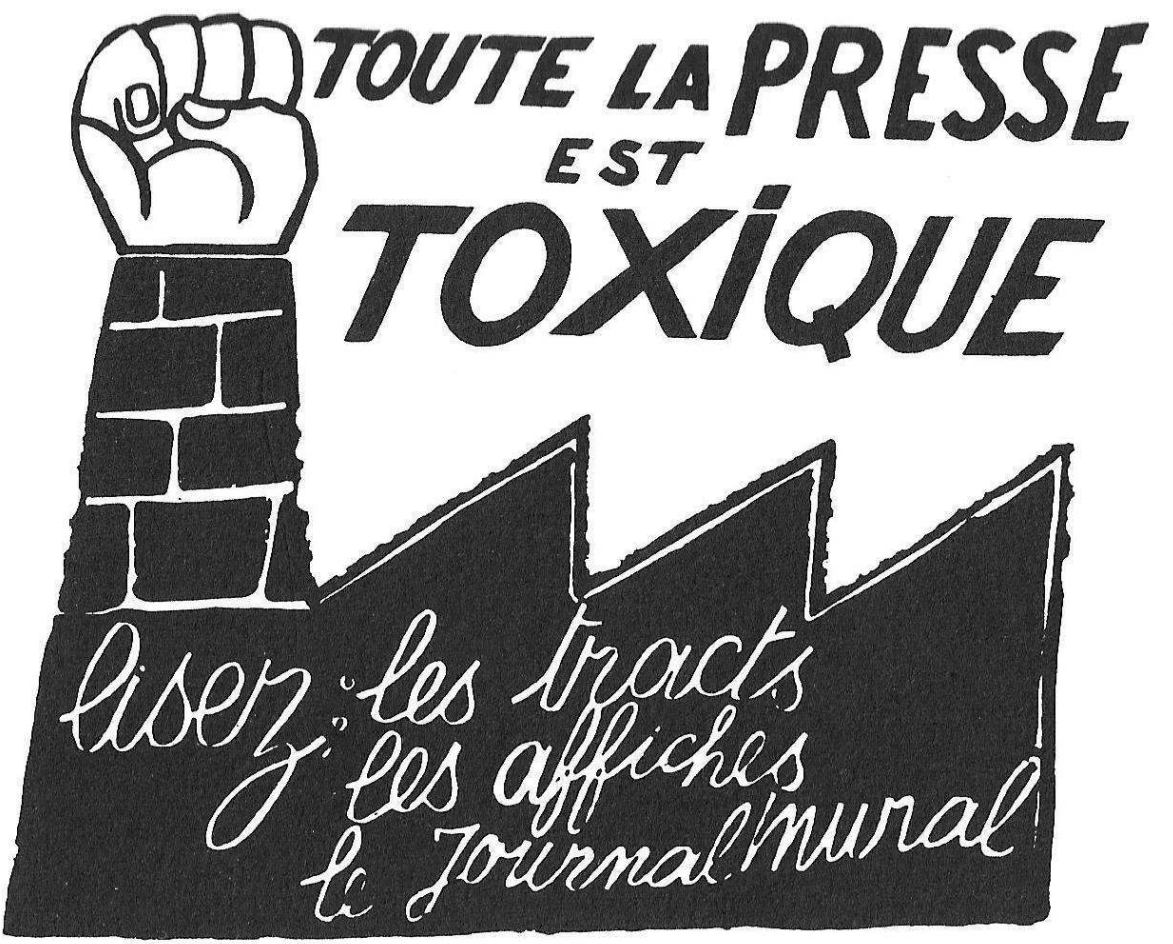

Illustration 16 : Toute la presse est toxique

Source : Gasquet, Vasco. 2007. 500 affiches de mai 68 (Bruxelles : Aden), p. 121 


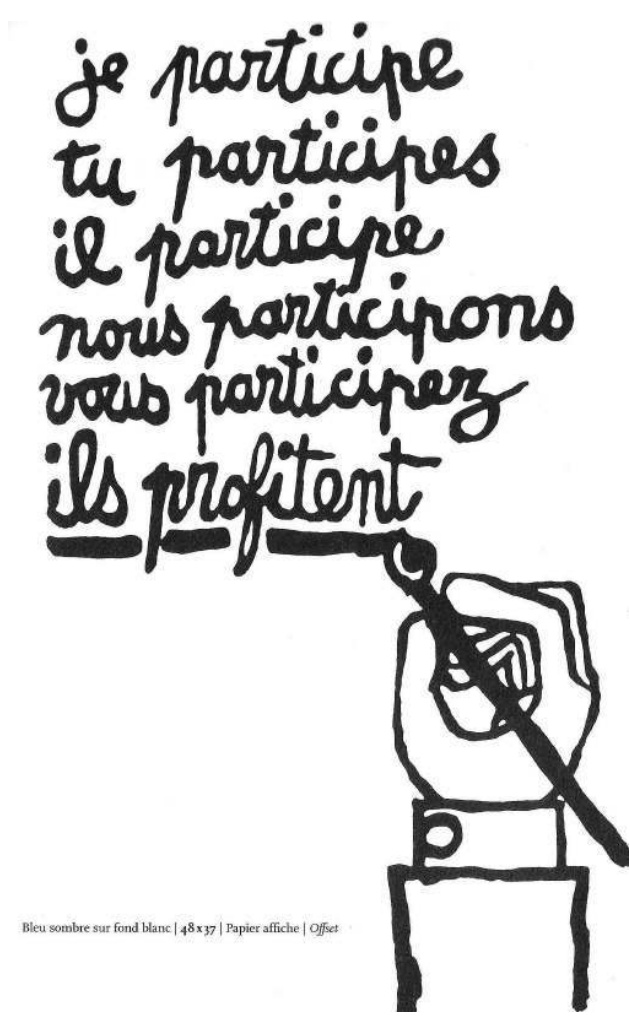

Illustration 17 : Je participe, tu participes

Source: Gasquet, Vasco. 2007. 500 affiches de mai 68 (Bruxelles : Aden), p. 55

Dans tous les cas, il y a là comme la proposition d'une alternative énonciative, qui entend faire de la production et de la manipulation libres des signifiants un geste hautement politique parce que non assignable à une identité sociale précise, là où l'argumentaire telquelien réserve le travail d'écriture révolutionnaire à la virtuosité des sujets théoriciens, dont l'identifiabilité sort renforcée. La subjectivation politique à l'œuvre dans les affiches procède non tant d'une opposition par rapport aux censeurs caricaturés, que d'une suspension de toute identification socio-discursive claire, au profit d'une appropriation des formes d'expression elles-mêmes.

L'instance énonciative qui assume cette appropriation, sans pour autant subir une typification socio-discursive, se donne la forme linguistique du «nous» - que l'on trouve dans les slogans "Nous sommes tous des Juifs et des Allemands", "Nous sommes tous des indésirables ", "Nous sommes un groupuscule », ou encore "À nous de parler ». La particularité de ce «nous » est que son référent ne préexiste pas à l'acte énonciatif, mais renvoie à une collectivité rêvée, innumérable, projetée comme résultante indéfinie de l'écriture/lecture réitérée des affiches. Ce que ces "nous " désignent n'est en effet guère interprétable à partir d'une lecture littérale des slogans, qui miment le procédé d'assignation identitaire de la parole du pouvoir, mais pour mettre en échec sa fonction ségrégative et stigmatisante au profit d'un processus ouvert d'appropriation énonciative. Tout sujet individuel qui s'engage dans l'écriture/ lecture de l'affiche ou du slogan est invité à assumer et à reconnaître l'émergence d'une communauté qui prétend se définir, d'abord et essentiellement, par l'acte même de sa prise de parole, ou plutôt par l'itération collective d'actes d'écriture/lecture singuliers ${ }^{24}$. 


\section{Conclusion et relance}

À partir de l'observation de deux massifs discursifs exactement contemporains, nous avons mis en évidence l'existence d'une topique commune, celle de la liberté d'expression comme libération de la production signifiante.

Le discours de Tel Quel dans Théorie d'ensemble saisit cette topique dans un argumentaire, dont le bouclage et la cohérence sont garantis par la référence au sujet de la théorie, agent de la libération qui s'instaure en nouvelle autorité en matière de gestion des forces signifiantes.

L'affiche militante de Mai 68 saisit quant à elle cette même topique dans une argumentation qui a pour enjeu fondamental la subjectivation politique des masses. Celle-ci s'opère par une dé-prise identitaire et une mise en connexion généralisée des signifiants.

En guise d'ouverture, on pourrait se demander si cette divergence dans la manière d'investir une même topique ne pointe pas également, plus généralement, une tension dans la définition de l'identité culturelle française. Ni les telqueliens, ni les affiches de Mai 68 ne thématisent très explicitement cette question de l'identité nationale; pourtant, chacun de ces massifs a connu une réception qui l'a attaché à une certaine idée de la France (la French Theory d'un côté, tout l'imaginaire entourant Mai 68 de l'autre). Quelle inscription les pratiques que nous avons examinées trouvent-elles dans un imaginaire marqué par la nationalité ?

La pratique de l'affichage de rue trouve certes sa source contemporaine dans le militantisme vietnamien (Ross 2010 : 147) et dans les dazibao, ces grands journaux muraux de la révolution culturelle maoïste (Calvet $1976: 102$ ), mais puise aussi à ce que Jacques Guilhaumou appelle la "gestuelle héroïque des Révolutions françaises" (Guilhaumou 2010: 170). On peut sans doute également la rattacher à l'abondante production de « parole ouvrière » entre 1830 et 1851, rassemblée sous ce titre par Alain Faure et Jacques Rancière (2007), qui témoigne déjà d'un travail sur le "sens social » des mots comme condition d'une émancipation collective ${ }^{25}$. Quant à la révolution textuelle prônée par Tel Quel, elle s'inscrit plutôt dans la tradition des avant-gardes esthétiques et de leurs tentations plus ou moins politiques.

L'intérêt de la conjoncture examinée est qu'elle fait coïncider chronologiquement, sur une même topique, les pratiques discursives de l'une et l'autre de ces traditions, de sorte à créer, vu de loin, un effet de cohérence. Si les travaux socio-historiques ont déjà pu montrer ce que cette cohérence avait de factice, nous espérons avoir pu en donner l'éclairage du point de vue rhétorique. Un dernier exemple nous servira de conclusion. Le collectif de Tel Quel pratiquera bien, lui aussi, l'affichage de dazibao, mais reproduits en tant que dazibao et uniquement dans les locaux du groupe ${ }^{26}$. Sorte de réplique du militantisme de l'affiche, désamorcée et déplacée en emblème, dans les bureaux de Tel Quel, la pratique du dazibao confirme la fascination du groupe pour ces usages $\mathrm{du}$ langage comme supports de la révolution, en même temps que son souci de récupérer ces formes en nouveaux attributs distinctifs de l'élite littéraire.

C'est peut-être là l'une des dialectiques qui caractérise l'histoire de la France moderne et participe à la fois de l'apparente unité et de la césure profonde de son identité culturelle : l'importation par l'élite lettrée des formats de la révolte des masses. 


\section{BIBLIOGRAPHY}

Angenot, Marc. 1989. 1889. Un état du discours social (Longueil : Le Préambule)

Barthes, Roland. 1968. « L'écriture de l'événement », Communications 12, 108-112

Boutet, Josiane. 2010. Le pouvoir des mots (Paris : La Dispute)

Calvet, Louis-Jean. 1976. La production révolutionnaire. Slogans, affiches, chansons (Paris : Payot)

Certeau, Michel de. 1968. La prise de parole. Pour une nouvelle culture (Paris : Desclée de Brouwer)

Faure, Alain \& Jacques Rancière (éds). 2007. La parole ouvrière 1830-1851 (Paris : La Fabrique)

Ffrench, Patrick. 1995. The time of theory: a history of Tel Quel (1960-1983) (Oxford : Oxford University Press)

Forest, Philippe. 1995. Histoire de Tel Quel (Paris : Seuil)

Forest, Philippe \& Patrick Ffrench (éds). 1999. De Tel Quel à L'Infini, l'avant-garde et après? (Nantes : Pleins feux)

Gasquet, Vasco. 2007. 500 affiches de mai 68 (Bruxelles : Aden)

Gobille, Boris. 2005. « Les mobilisations de l'avant-garde littéraire française en mai 1968. Capital politique, capital littéraire et conjoncture de crise ", Actes de la recherche en sciences sociales 158, 30-61

Gobille, Boris. 2008. Mai 68 (Paris : La Découverte)

Groupe $\mu$. 1977. Rhétorique de la poésie. Lecture linéaire, lecture tabulaire (Bruxelles : Complexe)

Guilhaumou, Jacques. 2010. « Mémoires d'un étudiant en mai 1968 : le flux des manifestations et le protagoniste de l'événement », Le Mouvement social 233, 165-181

Hutcheon, Linda. 2001. « Politique de l'ironie », Schoentjes, Pierre. Poétique de l'ironie (Paris : Seuil), 289-301

Jamoye, Marie-Céline. 2009. La révolution dans la société par l'écriture textuelle : l'utopie du métadiscours engagé de Tel Quel (1966-1968), Mémoire de maîtrise non publié, Université de Liège

Jenny, Laurent. 2008. Je suis la révolution. Histoire d’une métaphore (1830-1975) (Paris : Belin)

Kauppi, Niilo. 1994. The making of an avant-garde: Tel Quel (Berlin - New York : Mouton de Gruyter)

Klinkenberg, Jean-Marie. 2008. « La relation texte-image. Essai de grammaire générale », Bulletin de la Classe des Lettres. Académie royale de Belgique, $6^{\mathrm{e}}$ série, t. XIX, 21-79

Loyer, Emmanuelle. 2008. Mai 68 dans le texte (Paris : Complexe)

Pinto, Louis. 1991. « Tel Quel. Au sujet des intellectuels de parodie », Actes de la recherche en sciences sociales $89,66-78$

Rabatel, Alain. 2009. Homo narrans. Pour une analyse énonciative et interactionnelle du récit, vol. 1-2 (Limoges : Lambert-Lucas)

Rancière, Jacques. 2004. Aux bords du politique (Paris : Gallimard)

Roque, Georges. 1983. Ceci n'est pas un Magritte. Essai sur Magritte et la publicité (Paris : Flammarion) 
Roque, Georges. 2008. «Political Rhetoric in Visual Images », Weigand, Edda (éd.), Dialogue and Rhetoric (Amsterdam - Philadelphie : John Benjamins Publishing Company), 185-193

Roque, Georges. 2011. « Rhétorique visuelle et argumentation visuelle », Semen 32, 93-108

Ross, Kristin. 2006 [1996]. Rouler plus vite, laver plus blanc. Modernisation de la France et décolonisation au tournant des années 60 (Paris : Flammarion)

Ross, Kristin. 2010. Mai 68 et ses vies ultérieures (Marseille : Agone)

Tel Quel. 1968. Théorie d'ensemble (Paris : Seuil)

Tournier, Maurice. 2007. Les mots de mai 68 (Toulouse : Presses universitaires du Mirail)

Trebitsch, Michel. 2000. «Voyages autour de la révolution. Les circulations de la pensée critique, de 1956 à 1968 ", Dreyfus-Armand, Geneviève, Robert Frank, Marie-Françoise Lévy \& Michelle Zancarini-Fournel (éds), Les années 68. Le temps de la contestation (Paris : Complexe), 69-87

\section{NOTES}

1. Nous entendons ce terme au sens que lui a donné Marc Angenot, à la suite d'Aristote : « Il faut remonter à Aristote et appeler topique l'ensemble des "lieux" (topoï) ou présupposés irréductibles du vraisemblable social tels que tous les intervenants des débats s'y réfèrent pour fonder leurs divergences et désaccords parfois violents in praesentia, c'est-à-dire tout le présupposé collectif des discours argumentatifs et narratifs. [...] La topique produit l'opinable, le plausible, mais elle est aussi présupposée dans toute séquence narrative, elle forme l'ordre de véridiction consensuelle qui est condition de toute discursivité, qui sous-tend la dynamique d'enchaînement des énoncés de tous ordres " (Angenot 1989 : 28). Son usage ici au pluriel vise à en pointer le caractère potentiellement éclaté et donc dynamique.

2. Dans son recueil raisonné et commenté, Emmanuelle Loyer (2008) insiste sur cette même interprétation de la topique: "L'important n'est pas la liberté mais l'acte de libération et l'expérience concrète d'organisation, d'expression et de liberté qui s'invente, dans la plus grande improvisation au cours de ces semaines des mois de mai et de juin. [...] à l'odéon, la révolution se comprend surtout par la "communication" (un mot d'époque), la capacité du langage à faire lien et à transcender les fossés hérités des différences sociales » (2008: 163-164).

3. «Amnistie des yeux crevés ", comité d'action Nous sommes en marche, Censier, 13 mai 1968 ; cité dans Loyer $2008: 172$.

4. Tel que rassemblé par Gasquet (2007). Les illustrations sont reproduites ici avec l'aimable autorisation de Gilles Martin, directeur des éditions Aden. L'auteur tient à l'en remercier très chaleureusement.

5. Tels que rassemblés dans le collectif de Forest \& Ffrench (éds., 1999).

6. Le travail de Marie-Céline Jamoye (2009) propose une tentative intéressante de dépassement de cette alternative.

7. Sur cette activité et sur la conception collective de la création qui l'anime, voir également Loyer (2008 : 248-49).

8. Dorénavant, nous renverrons directement aux pages du volume Théorie d'ensemble (1968), en utilisant l'abréviation ThE.

9. L'auteur souligne notamment les décalages générationnels, les différences de capitaux économiques et culturels, la proximité ou l'éloignement avec l'orthodoxie du PCF, la rivalité au sein du champ restreint avec le dissident Jean-Pierre Faye et sa récente revue Change, pour expliquer la rupture de Tel Quel avec le CAEE (Comité d'action étudiants-écrivains révolutionnaires). Mené par Maurice Blanchot et Dionys Mascolo, ce comité représente le 
véritable point d'intersection entre le mouvement étudiant et l'avant-garde littéraire en Mai 68, tandis que «les membres de Tel Quel dénient au mouvement étudiant tout statut révolutionnaire » (Gobille $2005: 45)$.

10. On contrastera utilement cette analyse aux vues un peu différentes (et un peu hagiographiques) de Philippe Forest, qui suppose, quant à lui, que «étant donné son prestige parmi les étudiants [...] Tel Quel comptait des lecteurs assidus parmi les agitateurs de Nanterre ou ceux de la Sorbonne » (Forest $1995: 322$ ).

11. Dans un autre ouvrage consacré plus largement aux années 1960 françaises, Kristin Ross (2006) analyse finement des discours, mais qui relèvent dans leur grande majorité de la production littéraire.

12. On notera la frappante convergence de la comparaison utilisée: «La parole étudiante a débordé si pleinement, fusant de partout et s'inscrivant partout, que l'on aurait quelque droit à définir superficiellement - mais aussi peut-être essentiellement - la révolte universitaire comme une prise de la parole (comme on dit : prise de la Bastille) » (Barthes 1968 : 109) ; « En mai dernier, on a pris la parole comme on a pris la Bastille en $1789 »$ (Certeau $1968: 40$ ).

13. Voir les travaux de Georges Roque (notamment 2008 et 2011), qui défendent une conception dialogique et argumentative de la rhétorique visuelle et proposent des analyses dont s'est nourrie notre propre démarche.

14. Sur ces éléments, voir également Jenny (2008), en particulier le chapitre VII : «Tel Quel ou la révolution dans le texte ".

15. Sur ce slogan, considéré comme l'un des emblèmes du mouvement, voir Loyer 2008 : 77.

16. Cette manipulation littérale du matériau verbal dans le militantisme de Mai 68 n'est pas le propre de l'affiche. Maurice Tournier rapporte que «les métallos de Vénissieux démontent les lettres de BERLIET au-dessous de l'entrée de l'entreprise pour les replacer dans un ordre différent, celui de LIBERTE [...] ». (Tournier $2007: 69$ ).

17. C'est ce que Jean-Marie Klinkenberg (2008), dans sa grammaire des règles de l'« interaction verbo-iconique ", classe dans la catégorie des «relations morphologiques»: "les relations morphologiques entre texte et image mobilisent les fonctions iconiques de l'écriture. Dans ces fonctions, le tracé du signe graphique ou d'un bloc de signes graphiques renvoie iconiquement à un objet ou à un autre signe. Il y a là une interpénétration des signes de deux sémiotiques, que rend possible leur caractère spatial partagé. »Cet article fournit une gamme très complète et très précieuse d'outils d'analyse des relations verbo-iconiques, pour des corpus de tout type. Nous remercions très chaleureusement l'auteur pour avoir bien voulu nous en faciliter l'accès.

18. Voir à ce sujet les pages que Louis-Jean Calvet (1976) consacre aux affiches de Mai 68, dont s'inspirent également les présentes analyses.

19. Comme le précise justement Klinkenberg (2008), il n'y a jamais de redondance parfaite et complète entre les composantes verbales et iconiques d'un énoncé pluricodique. Ce que nous nommons ici « renforcement sémantique » renvoie aux cas où les contenus convergent.

20. Le néologisme allotopie est introduit par le Groupe $\mu$ dans leur Rhétorique de la poésie (1977), pour désigner tous les phénomènes de non-pertinence sémantique, ou rupture d'istotopie.

21. Voir Boutet (2010: 122-23), qui voit dans le défigement, l'intertextualité et la polysémie quelques-unes des principales techniques du discours de propagande militante. Calvet (1976: 112-113) pointe plus précisément, dans le corpus des affiches de Mai 68, le cas des affiches où le texte et l'image ont deux sens indépendants, mais dont le rapprochement produit un troisième sens, critique.

22. Voir à ce sujet Hutcheon 2001. Certes, ce mécanisme d'appel au déchiffrement est également exploité par le discours publicitaire (parfois antérieur à Mai 68, et parfois en résonnance étroite avec certains courants du surréalisme ; voir Roque 1983). La particularité des affiches de Mai 68 tient en bonne part à la grande simplicité technique de leur mode de production, qui d'une part a 
$\mathrm{pu}$ favoriser la collectivisation de la production, et qui d'autre part s'est traduite dans une stylistique spécifique et reconnaissable, assurant une réception elle aussi « virale ».

23. L'exigence de parler masqué s'éclaire, de manière très concrète, si l'on considère que la réaction de la police face à ces manifestations consiste évidemment à rétablir l'ordre social en cherchant à identifier les militants pour pouvoir les punir (Ross $2010: 100$ ).

24. Voir à ce propos le témoignage de Jacques Guilhaumou, qui voit dans «la perpétuelle confusion entre le "je" et le "nous" ", « le fait même de l'émancipation » (Guilhaumou 2010 : 173). $\mathrm{Au}$ moment où nous mettons la dernière main à cet article, en janvier 2015, l'attentat contre Charlie Hebdo suscite un émoi planétaire, qui donne une résonnance particulière à notre analyse. Nous avons vu en effet proliférer les occurrences des slogans «Je suis Charlie » / " Nous sommes tous Charlie» qui, bien qu'inscrits dans un contexte socio-idéologique tout différent, nous semblent illustrer un mécanisme socio-discursif comparable de dés-identification / subjectivation collective par accumulation itérative.

25. À ce sujet, voir aussi Boutet (2010: 171-72), dont l'analyse rejoint à bien des égards celle que nous avons menée ici : "L'émergence d'une expression ouvrière publique fut en phase avec les exigences critiques et réflexives sur la langue française que les révolutionnaires avaient érigées. Se mit ainsi en place, dans les discours ouvriers, l'exigence d'une réflexion sociale sur la langue, sur le signe et son économie ; une recherche de mots justes pour dire la condition ouvrière et les conditions de son émancipation. Pour dire la justice sociale, de nombreux discours publics ouvriers ont été construits sur une forme de subversion du sens communément admis des mots, c'est-à-dire, en fait, du sens admis, transmis et inculqué par les plus forts, par ceux qui ont le pouvoir (économique comme symbolique).»

26. Comme l'illustre le cliché reproduit en couverture de la monographie de Philippe Forest (1995) sur le groupe.

\section{ABSTRACTS}

The paper takes as its starting point one of the famous May 68 slogans, "Freedom of speech ", and considers it as a topic. I describe the rhetorical uses of this topic in two different sets of discourses, both carried by a collective voice: on the one hand the papers collected in Tel Quel's Théorie d'ensemble (1968), and on the other hand the activist posters released the same year. First, I highlight the common argument of Théorie d'ensemble. Secondly, I show how this argument also supports the rhetorical efficacy of some of the May 68 posters. Thirdly, I differentiate between the Tel Quel's argument and the posters' argumentation, especially with regard to the subjectivization effects carried on by these discourses.

L'article prend comme point de départ l'un des fameux slogans de Mai 68, "Libérez l'expression", pour le considérer comme une topique. Il en décrit alors les mises en œuvre rhétoriques dans deux corpus a priori distincts, mais ayant en commun le fait d'être portés par une parole collective : d'une part, le genre de la "Théorie », tel qu'il est investi collectivement dans le recueil Théorie d'ensemble de Tel Quel paru en 1968, et d'autre part le corpus des affiches militantes de la même année. Il s'agit d'abord de mettre en lumière ce qui constitue l'argumentaire commun aux diverses contributions individuelles du volume Théorie d'ensemble, et ensuite de voir comment cet argumentaire est aussi ce qui fonde l'efficace rhétorique de certaines affiches de Mai 68. Enfin, l'article démontre ce qui distingue l'argumentaire telquelien 
de l'argumentation des affiches, en particulier en ce qui concerne les effets de subjectivation dont ces discours sont porteurs.

INDEX

Mots-clés: discours de protestation, discours théorique, Mai 68, militantisme, subjectivation, Tel Quel (groupe)

Keywords: activism, May 68, protest discourse, subjectivation, Tel Quel (group), theoretical discourse

\section{AUTHOR}

\section{FRANÇOIS PROVENZANO}

Université de Liège 